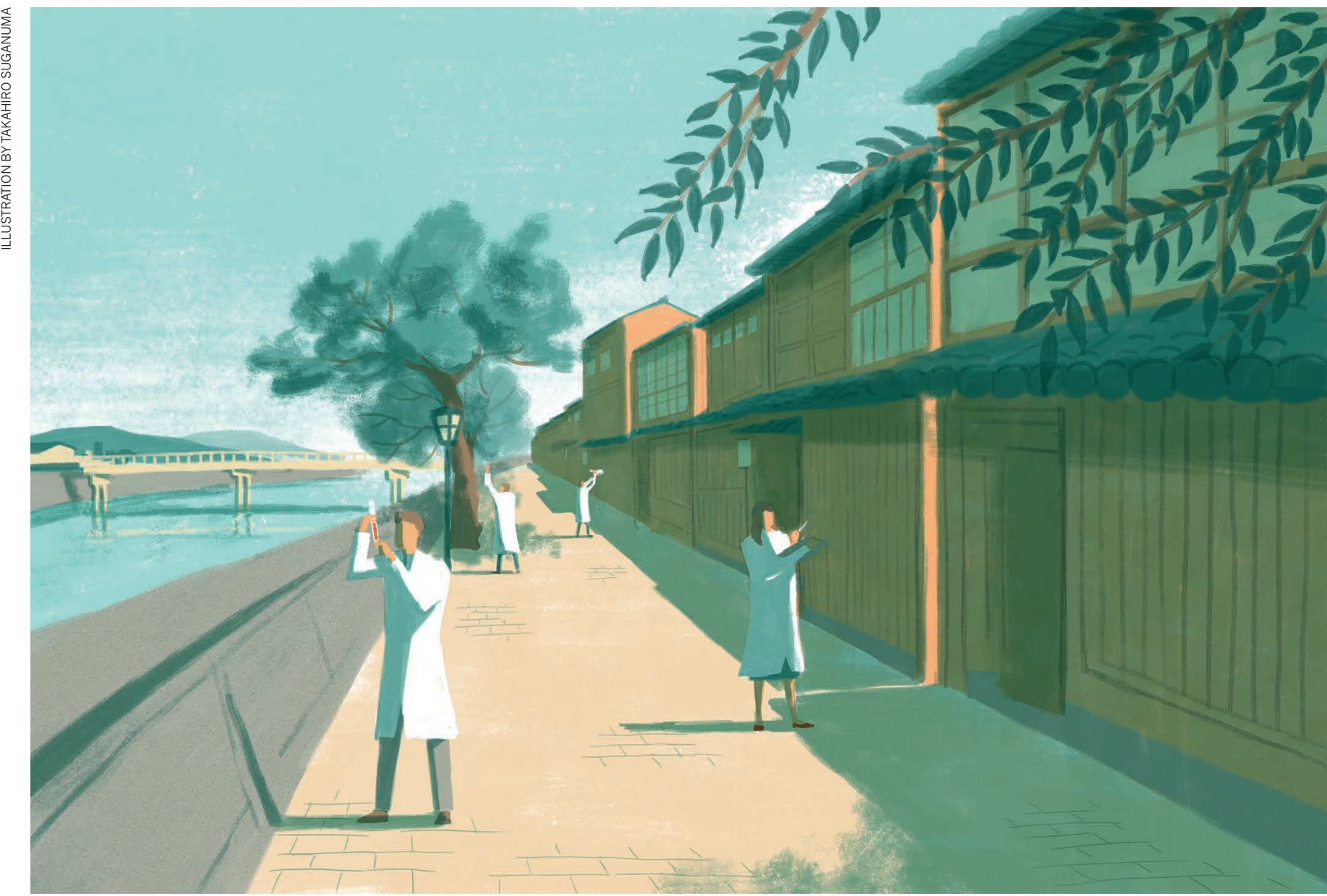

\title{
An alternative Japan experience
}

Kanazawa, on the Japan Sea Coast, is one city that most international researchers visiting the country never see and might make an attractive destination for scientists.

U ntil a few years ago, Kanazawa was relatively isolated on Japan's western coast, separated from the country's other population centres by the vastness of the Japanese Alps and the lack of a highspeed rail link. That changed in 2015, with the opening of the Hokuriku Shinkansen bullet-train service, which connects Kanazawa to Tokyo in two-and-a-half hours. This new accessibility has opened up Kanazawa, both as a tourist destination and as an alternative place to live and work outside Tokyo and its surrounding cities.

With a population approaching half a million, Kanazawa is not a big city, nor is it a country town. Wedged between the Alps and the Sea of Japan, and with pockets of historical neighbourhoods that have changed little since Japan's Edo period (1603-1868), the city makes for a very different experience from that found in the country's sprawling metropolises.

Kanazawa enjoys a healthy commercial sector, however. Tech giants Eizo and I-O Data have their headquarters nearby, and thanks to a boom in recent years, the area has one of the highest counts of information-technologyrelated offices and employees, per capita, in Japan.

The city is also home to three major research institutions: Kanazawa Institute of Technology, the Japan Advanced Institute of Science and Technology, and Kanazawa University (see 'Meet the big players'). Nature spoke to three researchers from these institutions about their life and work in Kanazawa.
NAKYOUNGCHONG Space and freedom to pursue research

Professor of information science, Japan Advanced Institute of Science and Technology (JAIST).

I moved to Kanazawa in 2003 to join JAIST as an associate professor. Before that, I was based in Tsukuba, north of Tokyo, at the National Institute of Advanced Industrial Science and Technology (AIST). I moved there from South Korea in 1998. One of my main reasons for moving to JAIST was 
the opportunity to pursue riskier or more innovative types of research than I could at AIST, but I've also found that the quiet location of the JAIST campus is exactly what I needed, because it gives me some insulation from the distractions of big cities such as Tokyo. I now have my own robotics laboratory, where I work as the Japan coordinator of an international collaboration aimed at developing the world's first culturally competent robot for elderly care as part of the culture-aware robots and environmental sensor systems for elderly support (CARESSES) project.

CARESSES aims to develop a culturally competent robot to address problems faced by ageing populations in the European Union and Japan. For my part, I am investigating a natural and intuitive way for robots to recognize human behaviour and understand human intent; this could be used for robots to acquire cultural knowledge about a user and to adapt their behaviour accordingly.

My research involves interactive experiments in a smart house at JAIST that is fully embedded with sensors and actuators for home automation, as well as testing and evaluation in an assisted-living facility. These experiments allow us to analyse the social and cultural aspects of elderly Japanese interacting with nursing-care robots in their daily lives.

Kanazawa is historically and culturally rich, and it feels calm compared with the major metropolitan areas of Japan. In Kanazawa, we are also surrounded on all sides by greenery and the sea. There is not much of an international community here yet, though, so not many international schools and the like. Foreigners are still quite rare here, so as a foreigner you will definitely stand out. For foreigners with kids, for example, their children are likely to be the only non-Japanese students in the classroom.
Despite this, I find many ways to collaborate internationally and to have an active role in technical professional organizations around the world. Since I joined JAIST, I've held several visitor and invited-professor positions in the United States, Europe and Korea. It helps being just 30 minutes from Komatsu International Airport.

\section{HERMINETERTRAIS Great research, immersed in Japanese culture}

\section{Company researcher, Innovative Composite Center (ICC), Kanazawa Institute of Technology.}

I am a researcher and $\mathrm{PhD}$ candidate with the ESI Group, a leading international innovator in virtual prototyping software, and I am normally based in France. I am currently part of a project being undertaken as a partnership between the ESI Group and the ICC, which has advanced experimental devices for compositematerial processing that will provide us with the essential data we need to develop a realtime virtual-prototyping simulation tool called Hybrid Twin.

Before I arrived in Kanazawa this May, I spent April in Tokyo at the ESI office there. It was a great introduction to the work environment and culture in Japan, because it was my first time in the country.

Kanazawa is a lively city with tourist hotspots, but it manages to keep its authenticity. I

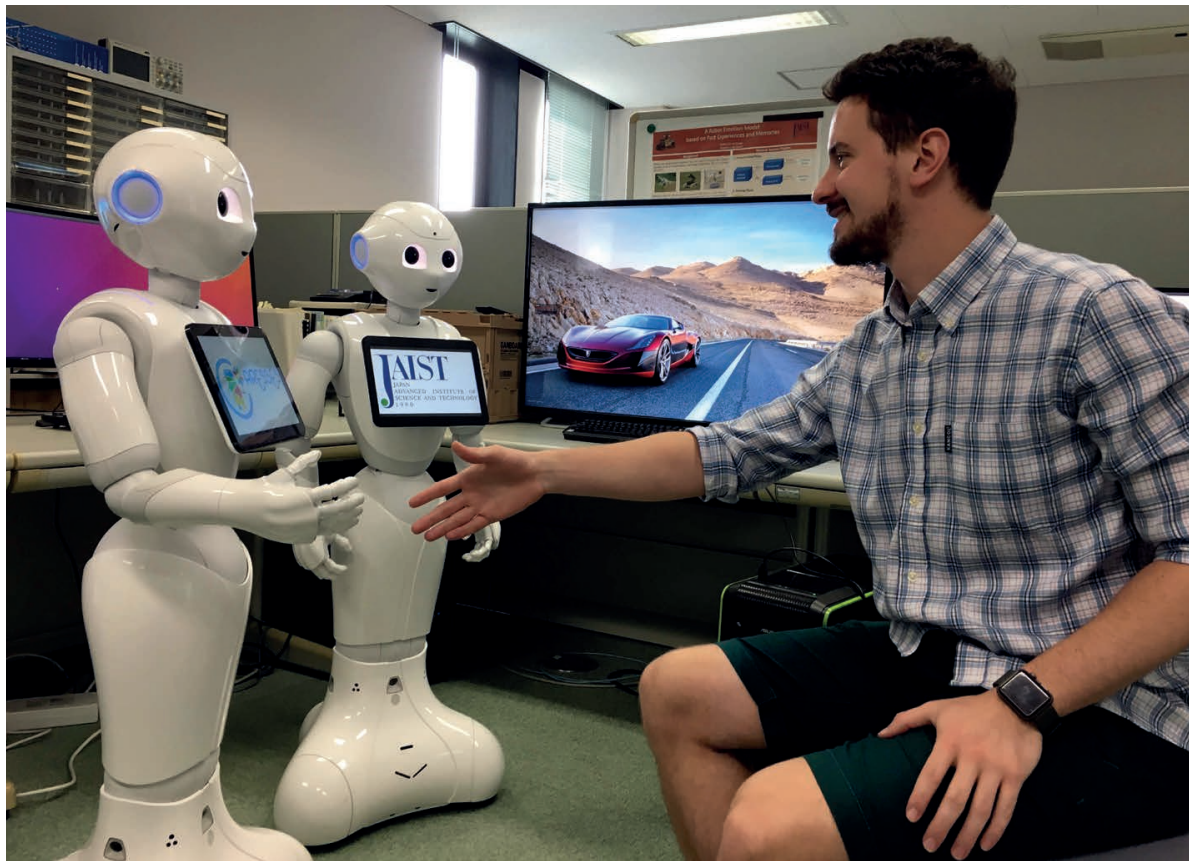

Nak Young Chong is working to develop robots in health care. really appreciate that the city centre is compact and everything is within walking distance, but you can drive for an hour and be in the mountains, on the beach or in the middle of rice fields. Compared with Tokyo, I have found that there is plenty of entertainment in Kanazawa, but the city is much less crowded. Language is a major obstacle here for foreigners, however. Many of my co-workers in Tokyo could speak some English; that's not the case here in Kanazawa, and so I do not have many exchanges with other researchers. In my project, I work mainly with the international network I had before coming here, but I have not been here long. The ICC is also quite new, and it feels like there is not yet much of an international scientific community here or throughout Kanazawa.

I found the research environment at the Nihon ESI headquarters in Tokyo in many ways comparable to research centres in France. As a woman in mechanical engineering, I am used to a male-dominated environment. Although that was the case in Tokyo, there were also a number of women in technical positions, and many could speak some English. Here at the ICC, however, there are no other female researchers; this, along with my limited Japanese-language proficiency, has limited my communication with the rest of the ICC team.

The research I am conducting here with ESI is very exciting. I'm working at an intersection between composite-material processing and advanced numerical techniques. The different projects I am working on aim to create or improve innovative simulation tools for industries in which composite materials are used - mainly aeronautic and automotive industries. The ICC has state-of-the-art technologies and machines that are used in composite material engineering. Living in Kanazawa is a real immersion in Japanese culture among very welcoming people.

\section{ROBERT JENKINS A place and an education to be proud of}

\section{Assistant professor of palaeontology and Earth science, Kanazawa University.}

I joined Kanazawa University five years ago. Previously, I was in Tokyo. The first time I visited Kanazawa was to interview for my current position, even though I am myself Japanese and have lived in Japan for most of my life. Although Kanazawa is somewhat isolated compared with Tokyo, Osaka and even Nagoya, it is actually not far from those cities. Further, I believe that researchers' ability to concentrate is actually enhanced by 


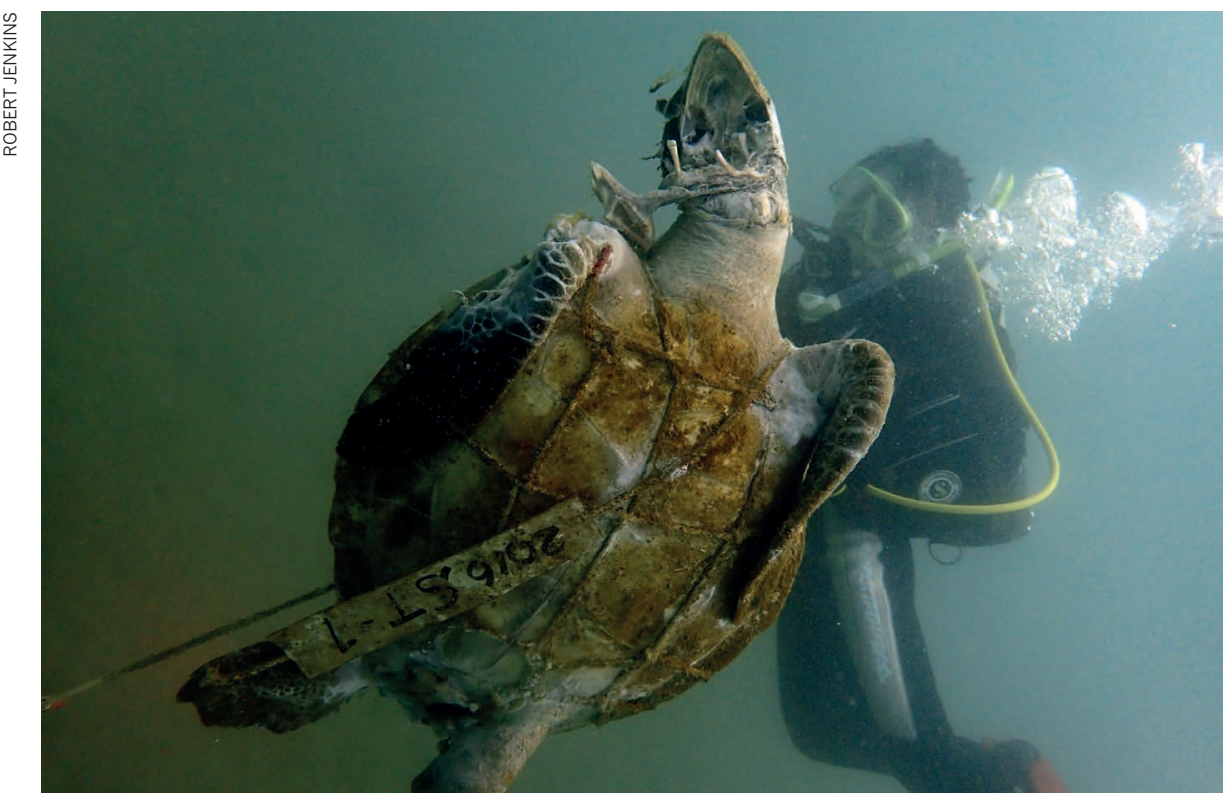

Large pieces of organic matter falling to the sea floor can form complex ecological environments.

Kanazawa's moderate level of isolation and its proximity to nature - the Sea of Japan, the Alps and natural wilderness are all within a short distance from the city centre.

The level of education here is very high, and people are proud of the fact that elementary and junior-high-school students from the area often rank first or second in national examinations. This is also reflected in Kanazawa's number of universities per capita, which is the third highest in Japan. People here like 'academic' things and appreciate the importance of science, so the atmosphere is fantastic both for conducting research and for living here as a researcher.

I think for foreigners, in particular, Kanazawa is a special place in Japan, where the old town and traditional customs such as tea ceremonies are very well preserved. Kanazawa City is one of the few major cities that were spared bombing during the Second World War, and so many traditions and much of the old architecture have survived.
I am researching the evolution of animals living in extreme environments, for example, near hydrothermal vents and cold seeps in the geologically active areas of the deep sea. The hypothesis I am working on is that such animals might have adapted from the communities that form near decaying organic matter on the sea floor, such as 'whale falls'. Last year, we reported on fossil sea-turtle remains from 80 million to 90 million years ago, long before the emergence of whales. I am lucky because Kanazawa University's marine station is located nearby on the Noto Peninsula, which, although just 50 kilometres or so from Kanazawa, is remote enough to allow us to study decaying processes. And for my work, the Japanese archipelago is very interesting, being one of the most geologically active areas in the world. I think research from Kanazawa will be able to shed light on the evolution of life on Earth.

INTERVIEWS BY BRETT DAVIS

Interviews have been edited for clarity and length.

\section{MEET THE BIG PLAYERS The three major research institutes in Kanazawa}

Kanazawa Institute of Technology is a private higher-learning institute with more than 8,000 students and close to 350 teaching staff. It is known for its advanced mechanical-engineering facilities and workshops.

Japan Advanced Institute of Science and Technology is a postgraduate education and research institution. About $40 \%$ of its more than 1,100 students and $20 \%$ of its 150 faculty researchers are from overseas. Most foreign researchers obtain their positions by applying for an associate professor or five-year assistant professor position.

Kanazawa University is a prominent university on the Sea of Japan coast with more than 10,000 students and 1,000 teaching and faculty staff members. It has been active in increasing the number of international students and students studying abroad over the past two or three years, has developed collaborative and exchange relationships with dozens of universities worldwide and has introduced Englishlanguage programmes for students and faculty and staff members. B.D. 


\section{CORRECTION}

The Spotlight article 'An alternative Japan experience' (Nature 562, S53-S55; 2018) incorrectly stated that Nak Young Chong joined JAIST as a visiting professor. In fact, he was jointly appointed as associate professor at both JAIST and AIST. 